\title{
Measuring of False Peaks Occurring via Planar Antenna Arrays DOA Estimation
}

\author{
Yuri B. Nechaev and Ilia W. Peshkov
}

\begin{abstract}
Direction-of-arrival (DOA) of radio signals takes a great scientific interest for radar, sonar and wireless communication tasks. In this paper the problem of DOA estimation methods for circular and concentric circular antenna arrays is considered. The super-resolution method MUSIC is researched. The performances are estimated in various noise environments and for various geometries of antenna arrays. Additionally, the problem of false peaks occurring in spatial spectrum is closely considered. Probability of occurring false peaks after computer simulations is presented.
\end{abstract}

Keywords - antenna array; circular arrays; concentric circular arrays; DOA estimation; super resolution.

\section{INTRODUCTION}

DOA-estimation of signal sources takes an important interest for such tasks as radars, sonars and wireless communications. DOA-estimation is crucial for radar technologies to detect and divide several signal sources, while in wireless communication systems information about signal coordinates allows spatial multiplexing to increase the number of simultaneously working users [1].

In that kind of application, spatial processing methods require the output signals from all the antenna elements to be in the digital domain. The output signals are necessary to be processed simultaneously. Increasing the number of transceiver units levels up output signal-noise ratio (SNR). It is known that high SNR value indeed enhances accuracy and spatial resolution of the DOA-estimation methods [2]. At present there are a lot of DOA-estimation methods which are capable to resolute two close signals located in one resolution interval according to the Rayleigh rule. Most of them can be related to subspace projection ones (as MUSIC, ESPRIT), their comprehensive description is given in [1-4]. The algorithms were investigated very closely for linear and circular antenna arrays (CAR) [3, 4]. Additionally, it is known that geometry of the antenna arrays may bound the DOA-estimation performances [5-7]. In particular the most important drawback of the linear antenna array is the only azimuth scanning. The planar antenna array was offered to

Manuscript received October 27, 2016, revised May 03, 2017

The reported study was funded by RFBR according to the research project No. 16-37-00072.

$\mathrm{Yu}$. B. Nechaev is with the Information Systems dept., Voronezh State University, Voronezh, Russia.

I. W. Peshkov, is with the Radio electronics and Computer Science dept. Yelets State University, Yelets, Russia (e-mail: ilvpeshkov@gmail.com).

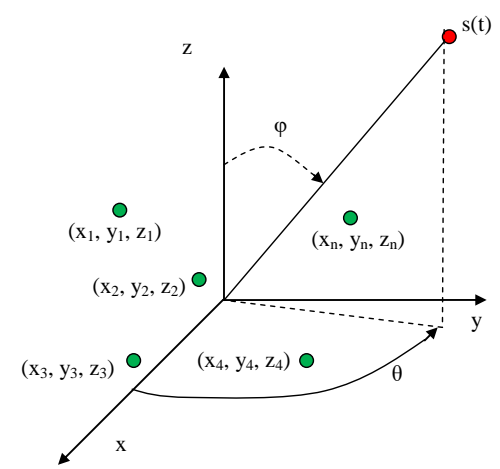

Fig. 1. Antenna array of arbitrary form. Red - signal source, Green - antenna elements

overcome the problem of the only azimuth direction finding. It can be used as azimuth as elevation direction finding. Moreover, circular and concentric circular arrays (CCA) were offered to scan in the two dimensions [8].

\section{MAIN DEFINITIONS}

Fig. 1 shows an arbitrary array consisting of $N$ antenna elements randomly arranged in space. Considering a narrowband signal $s(t)$ on carrier frequency $\omega_{0}$ having angular coordinates $\theta$ and $\phi$ relative to axes $x$ and $z$ respectively. Note that $\theta$ is an azimuth coordinate and $\phi$ is an elevation one. The signal can be expressed as [9]:

$$
\tilde{s}(t)=u(t) \cos \left\{\omega_{0}+v(t)\right\}
$$

As we can see from fig. 1, the delay depends on relative position of antenna elements and angular coordinates of the signals. If we take the origin as the reference point and $i$ th element has coordinates $\left(x_{i}, y_{i}, z_{i}\right)$, then the delay $\tau_{i}$ of the signal at $i$ th element relative to the origin can be expressed as [9]:

$$
\tau_{i}=c^{-1}\left[x_{i} \sin \varphi \cos \theta+y_{i} \sin \varphi \cos \theta+z_{i} \cos \varphi\right]
$$

As long as the signal is narrowband, then the delay $\tau_{i}$ produces the phase shift $\xi_{i}=-\tau \omega_{0}$, that is

$$
\begin{gathered}
s(t-\tau)=s(t) e^{j \xi_{i}}=s(t) e^{-j \tau_{i} \omega_{0}} \\
\xi_{i}=\frac{\omega_{0}}{c}\left[x_{i} \sin \varphi \cos \theta+y_{i} \sin \varphi \cos \theta+z_{i} \cos \varphi\right]
\end{gathered}
$$

where $\lambda$ is wavelength, $\omega_{0} / \mathrm{c}=2 \pi / \lambda$. And now, the signal at the array outputs can be described in the vector form as: 


$$
\begin{gathered}
\mathbf{x}(t)=\left[\begin{array}{llll}
e^{j \xi_{1}} & e^{j \xi_{2}} & \cdots & e^{j \xi_{N}}
\end{array}\right]^{T} s(t)=\mathbf{a}(\omega, \theta, \lambda) s(t) \\
\mathbf{a}(\omega, \theta, \lambda)=\left\lfloor\begin{array}{llll}
e^{j \mathbf{k}_{1}^{T}} & e^{j \mathbf{k}_{2}^{T}} & \cdots & e^{j \mathbf{k}_{N}^{T}}
\end{array}\right]
\end{gathered}
$$

where $\quad \mathbf{k}=\frac{2 \pi}{\lambda}\left(k_{x}, k_{y}, k_{z}\right)=(\sin \varphi \cos \theta, \sin \varphi \sin \theta, \cos \varphi)$

wave number, $\mathbf{r}_{n}^{T}=\left(x_{n}, \mathrm{y}_{n}, \mathrm{z}_{n}\right)^{T}$ is the radius-vector pointed to the $n$-th antenna element.

For an arbitrary geometry configuration antenna array a complex output signal vector can be written as:

$$
\overrightarrow{\mathbf{x}}(t)=\mathbf{A} \cdot \overrightarrow{\mathbf{s}}(t)+\overrightarrow{\mathbf{n}}(t),
$$

where $\overrightarrow{\mathbf{x}}(t)$ - describing output signals vector, $\overrightarrow{\mathbf{s}}(t)$ - signal vector, $\overrightarrow{\mathbf{n}}(t)$ - noise vector, A - matrix of steering vectors, $m$ th column of the matrix describes phase distribution of $m$ th signal source inside antenna array.

Let's assume, that $\overrightarrow{\mathbf{s}}(t)$ and $\overrightarrow{\mathbf{n}}(t)$ are stationary random processes, $\overrightarrow{\mathbf{n}}(t)$ is the Gaussian random process with zero mean and a covariance matrix $\sigma^{2} \mathbf{I}\left(\sigma^{2}\right.$ - noise variance) and that the signals are uncorrelated and there is no correlation between noise and signals. Then spatial correlation matrix can be written in the following form:

$$
\mathbf{R}=E\left[\overrightarrow{\mathbf{x}}(t) \overrightarrow{\mathbf{x}}^{H}(t)\right]=\mathbf{A S} \mathbf{A}^{H}+\sigma^{2} \mathbf{I},
$$

where $\mathrm{E}[\ldots]$ is the mathematical expectation, $(\ldots)^{\mathrm{H}}$ is the Hermitian transpose, $\mathbf{S}=E\left[\overrightarrow{\mathbf{s}}(t) \overrightarrow{\mathbf{s}}^{H}(t)\right]$ is the signal correlation matrix.

The output signal is generated as a sum of several uncorrelated external signal sources and the thermal noise. In this case we get:

$$
\overrightarrow{\mathbf{x}}(t)=\sum_{m=0}^{M-1} s_{m}(t) \overrightarrow{\mathbf{a}}\left(\theta_{m}\right)+\overrightarrow{\mathbf{n}}(t) .
$$

If the distinct signals are uncorrelated, the correlation matrix is as a sum of distinct correlation matrices of the signals and the noise:

$$
\begin{aligned}
& \mathbf{R}=\sum_{m=0}^{M-1} E\left[s_{m}^{2}(t)\right] \overrightarrow{\mathbf{a}}^{H}\left(\theta_{m}\right) \overrightarrow{\mathbf{a}}\left(\theta_{m}\right)+\sigma_{0}^{2} \mathbf{I}= \\
& =\sum_{m=0}^{M-1} p_{m} \overrightarrow{\mathbf{a}}^{H}\left(\theta_{m}\right) \overrightarrow{\mathbf{a}}\left(\theta_{m}\right)+\sigma^{2} \mathbf{I}=\mathbf{A S S} \mathbf{A}^{H}+\sigma^{2} \mathbf{I}
\end{aligned}
$$

where $\mathbf{S}$ - signals correlation matrix, $p_{i}$ - power of $i$ th signal.

As the rank of matrix $\mathbf{A S A}{ }^{H}$ is equal to $M$, then $\mathbf{A S A}{ }^{H}$ has $M$ positive eigenvalues, and the rest $(N-M)$ ones are equal to zero. Let $\lambda_{1} \geq \lambda_{2} \geq \ldots \geq \lambda_{N-1} \geq \lambda_{N}$ be eigenvalues of the correlation matrix $\mathbf{R}$. Let $\mathbf{E}_{\mathrm{s}}=\left[\overrightarrow{\mathrm{e}}_{1}, \overrightarrow{\mathrm{e}}_{2}, \ldots, \overrightarrow{\mathrm{e}}_{M}\right]$ orthonormal eigenvectors related to the $M$ biggest eigenvalues $\Lambda_{\mathrm{s}}=\left[\lambda_{1}, \lambda_{2}, \ldots, \lambda_{\mathrm{M}}\right]$ and $\mathbf{E}_{\mathrm{n}}=\left[\overrightarrow{\mathrm{e}}_{\mathrm{M}+1}, \overrightarrow{\mathrm{e}}_{\mathrm{M}+2}, \ldots, \overrightarrow{\mathrm{e}}_{\mathrm{N}}\right]$ be the matrix consisting of orthonormal eigenvectors related to the $(N-M)$ smallest eigenvalues $\Lambda_{n}=\left[\lambda_{\mathrm{M}+1}, \lambda_{\mathrm{M}+2}, \ldots, \lambda_{\mathrm{N}}\right]$.

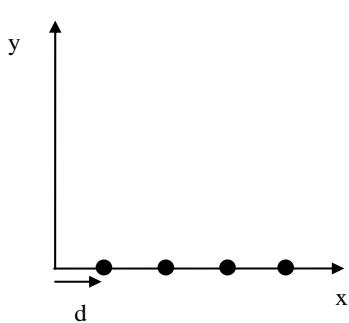

(a)

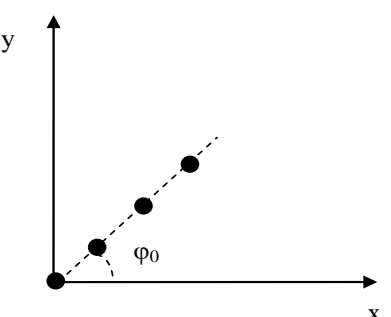

(b)
Fig. 2. a) antenna moving, b) antenna rotating Let us assume that [10]:

$$
\mathbf{R E}_{n}=\Lambda_{n} \mathbf{E}_{n}=\sigma_{0}^{2} \mathbf{E}_{n}=\mathbf{A S} \mathbf{A}^{H} \mathbf{E}_{n}+\sigma^{2} \mathbf{E}_{n},
$$

then it means, that $\mathbf{A}^{H} \mathbf{E}_{n}=0$. As the eigenvectors of the noise subspace are orthogonal to the columns of the steering matrix $\mathbf{A}$, then the property matches the true coordinates of the signal sources $\left\{\phi_{m}, \theta_{m}\right\}_{m=0}^{M-1}$. Spatial spectrum of MUSIC is as [11]:

$$
P_{M U S I C}(\phi, \theta)=\left[\overrightarrow{\mathbf{a}}^{H}(\theta) \mathbf{E}_{N} \mathbf{E}_{N}^{H} \overrightarrow{\mathbf{a}}(\theta)\right]^{-1}
$$

The numerator of (2) is equal to zero and function $P_{\text {MUSIC }}(\phi, \theta)$ goes to infinity relating to the true $\left\{\hat{\phi}_{m}, \hat{\theta}_{m}\right\}_{m=0}^{M-1}$.

\section{PlanAR ANTENNA ARRAYS}

If an antenna array is not placed at the origin and starts from the origin at $d$, as show in fig. 2, then an array factor is [12]:

$$
A F_{s}=e^{j \Psi}+e^{j 2 \Psi}+\ldots+e^{j N \Psi}=e^{j \Psi} \sum_{n=0}^{N} e^{j n \Psi}=e^{j \Psi} A F
$$

If an antenna array is rotated at angle $\varphi 0$ (fig. $2 b$ ), then its array factor gets form [12]:

$$
A F_{r}=\sum_{n=0}^{N} e^{j n[k d \sin \phi \cos (\theta-\varphi 0)+\beta]} .
$$

In order to calculate a planar array's factor $A F p$, it is possible to make several combinations of rotated and moved copies of the linear antenna arrays. If the linear array's factor placed along the $x$ is designated, then by using the properties of the moved linear arrays (3) and (4) and combining them we can get an array factor of any shape array.

The steering vectors and array factors are necessary to apply direction-finding tasks. However, the equation of a hexagonal arrays' factor is very complicated [13], whereas an octagonal one has not been found at all. We can say that an arbitrary antenna array is a summation of several moved and rotated linear arrays. Let us consider building a hexagonal array out of 12 antenna elements. Provided that the first side consists of a linear antenna array and located along the $x$, its factor will:

$$
A F_{1}=\sum_{n=0}^{N-1} e^{j k_{x} n d}
$$

Here $N=2$ is the number of antenna elements along $x$ direction. The linear antenna array should be rotated at $60^{\circ}$ 
and moved by $2 d$ in order to calculate the second side's factor. Using the properties (3) and (4), the second side's factor is set as

$$
A F_{2}=e^{j k} 4 d \sum_{n=0}^{N-1} e^{j k n d \sin \varphi \cos (\theta-\pi / 3)}
$$

Side 3 is rotated at $120^{\circ}$ and after that moved by the distance $2 d$ in the direction $30^{\circ}$ about the $x$ axis in the same way.

$$
A F_{3}=e^{j\left(k_{x}(1+\cos \{\pi / 3\})+k_{y} \sin \{\pi / 3\}\right) 4 d} \sum_{n=0}^{N-1} e^{j k n c \sin \varphi \cos (\theta-2 \pi / 3)}
$$

The similar actions are carried out for the rest of the sides. As a result we get the hexagonal antenna array factor (AFHEX):

$$
\begin{gathered}
A F_{4}=e^{j\left(k_{x}+2 k_{y} \sin \{\pi / 3\}\right) 4 d} \sum_{n=0}^{N-1} e^{j k n d \sin \varphi \cos (\theta-\pi)} \\
A F_{5}=e^{j\left(2 k_{y} \sin \{\pi / 3\}\right) 4 d} \sum_{n=0}^{N-1} e^{j k n d \sin \varphi \cos (\theta-4 \pi / 3)} \\
A F_{6}=e^{j\left(-k_{x} \cos \{\pi / 3\}+k_{y} \sin \{\pi / 3\}\right) 4 d} \sum_{n=0}^{N-1} e^{j k n n \sin \varphi \cos (\theta-5 \pi / 3)}, \\
A F_{H E X}=\sum_{p=0}^{5} A F_{(p)}
\end{gathered}
$$

So using the approach helps to derive the octagonal array's factor $\left(A F_{O C T}\right)$ [14]:

$$
\begin{aligned}
& A F_{O C T}=A F_{1}+ \\
& e^{j k_{x} 3 d} A F_{l}(\pi / 4)+ \\
& e^{j\left(k_{x}(1+\cos \{\pi / 4\})+k_{y} \sin \{\pi / 4\}\right) 3 d} A F_{l}(2 \pi / 4)+ \\
& e^{j\left(k_{x}(1+\cos \{\pi / 4\})+k_{y}(1+\sin \{\pi / 4\})\right) 3 d} A F_{l}(3 \pi / 4)+ \\
& e^{j\left(k_{x} \cos \{\pi / 4\}+k_{y}(1+2 \sin \{\pi / 4\})\right) 3 d} A F_{1}(\pi)+ \\
& e^{j\left(k_{y}(1+2 \sin \{\pi / 4\})\right) 3 d} A F_{1}(5 \pi / 4)+ \\
& e^{j\left(-k_{x} \cos \{\pi / 4\}+k_{y}(1+\sin \{\pi / 4\})\right) 3 d} A F_{l}(6 \pi / 4)+ \\
& e^{j\left(-k_{x} \cos \{\pi / 4\}-k_{y} \sin \{\pi / 4\}\right) 3 d} A F_{l}(7 \pi / 4)
\end{aligned}
$$

By that way, it is very easy to derive array factor of an antenna array of any shape.

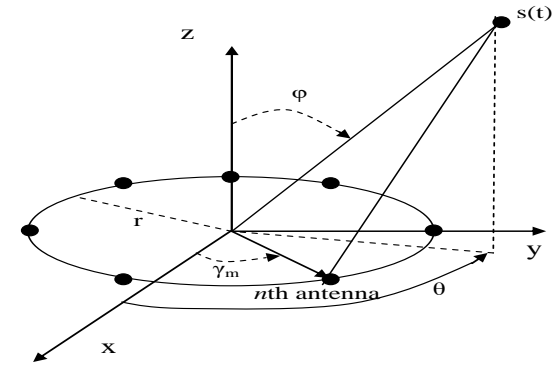

Fig. 3. Circular antenna array

\section{CIRCUlar AND CONCENTRIC ARRAYS}

In fig. 3 the CAR with radius $r$, consisting of $N$ isotropic elements is depicted. The $n$th value of the steering vector $\overrightarrow{\mathbf{a}}(\phi, \theta)$ describing phase shifts of the signal arriving at the antenna array from direction $\theta-$ in azimuth and $\varphi-$ elevation space in the Euclidean coordinate space, and can be written [8]:

$$
a_{n}^{\operatorname{circ}}\left(\phi_{m}, \theta_{m}\right)=e^{\left\{j\left[-k_{m} r \cos \left(\theta_{m}-\frac{2 m n}{N}\right) \sin \left(\phi_{m}\right)\right]\right\}},
$$

where $r$ is the radius; $n=1 \ldots N, k_{m}=2 \pi / \lambda_{m}, \lambda_{m}$ is the wavelength of $m$ th signal source.

The geometry of concentric antenna arrays is built by means of several circular antenna arrays placed one into another.

In fig. $4 H$ circular antenna arrays are depicted. Each antenna array has $K_{h}$ antenna elements, where index $h$ represents the number of corresponding circular array $1 \leq h \leq H$. The circular antenna array can own different numbers of elements in its construction, so then it can be formed in different geometries. In the structure of each circular array $r_{h}$ is the radius of $h$ th circular array and the angle between adjacent elements is equal to:

$$
\gamma_{k_{h}}=2 \pi \frac{k_{h}}{K_{h}}
$$

where $k_{h}=0,1, \ldots, K_{h^{-}} 1$ and $h=1,2, \ldots, H$. So that, the phase in $k_{h}$ th element of $h$ th circular antenna array can be written:

$$
a_{n}^{\text {circ }}\left(\phi_{m}, \theta_{m}\right)=e^{\left\{j\left[-k_{m} r_{h} \cos \left(\theta_{m}-2 \pi \frac{k_{h}}{K_{h}}\right) \sin \left(\phi_{m}\right)\right]\right\}}
$$

Then the steering vector of $m$ th signal source at $h$ th circular antenna array in terms of azimuth and elevation angles and the position of the each element in the space takes the form:

$$
\mathbf{a}_{h}\left(\phi_{m}, \theta_{m}\right)=\left[\begin{array}{c}
e^{\left\{j\left[-k_{m} r_{h} \cos \left(\theta_{m}-\gamma_{0}\right) \sin \left(\phi_{m}\right)\right]\right\}} \\
e^{\left\{j\left[-k_{m} r_{h} \cos \left(\theta_{m}-\gamma_{1}\right) \sin \left(\phi_{m}\right)\right]\right\}} \\
\cdots \\
e^{\left\{j\left[-k_{m} r_{h} \cos \left(\theta_{m}-\gamma_{K_{h}-1}\right) \sin \left(\phi_{m}\right)\right]\right\}}
\end{array}\right]
$$

The matrix of the steering vectors of $h$ th CAR is written as:

$$
\mathbf{A}_{h}=\left[\begin{array}{llll}
\mathbf{a}_{h}\left(\phi_{0}, \theta_{0}\right) & \mathbf{a}_{h}\left(\phi_{2}, \theta_{2}\right) & \cdots & \mathbf{a}_{h}\left(\phi_{M-1}, \theta_{M-1}\right)
\end{array}\right]
$$

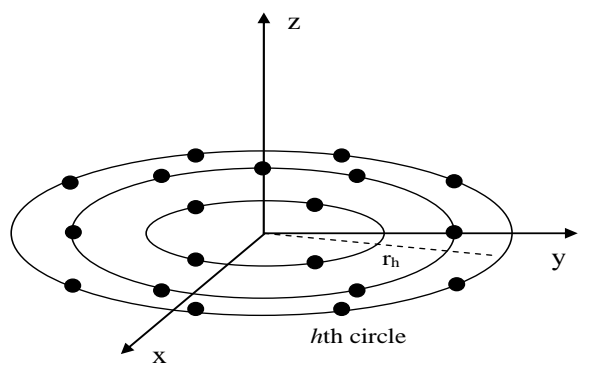

Fig. 4. Concentric antenna array 
The matrix of the steering vectors of entire concentric antenna array can be defined as a combination of all the matrices of the steering vectors of each circular array with common phase center as a reference [8]:

$$
\mathbf{A}=\left[\begin{array}{llll}
\mathbf{A}_{1} & \mathbf{A}_{2} & \cdots & \mathbf{A}_{H}
\end{array}\right]^{T} .
$$

In the case of $M$ signal sources and the entire number of antenna elements $N=\left(K_{l}+K_{2}+\ldots+K_{H}\right)$, the signal model at the output of the concentric antenna array remains similar to (1). The matrix of the steering vectors can be written in a more appropriate way [8]:

$$
\mathbf{A}=\left[\begin{array}{cccc}
\mathbf{a}_{0}\left(\phi_{0}, \theta_{0}\right) & \mathbf{a}_{0}\left(\phi_{1}, \theta_{1}\right) & \cdots & \mathbf{a}_{0}\left(\phi_{M-1}, \theta_{M-1}\right) \\
\mathbf{a}_{1}\left(\phi_{0}, \theta_{0}\right) & \mathbf{a}_{1}\left(\phi_{1}, \theta_{1}\right) & \cdots & \mathbf{a}_{1}\left(\phi_{M-1}, \theta_{M-1}\right) \\
\vdots & \vdots & \vdots & \vdots \\
\mathbf{a}_{H}\left(\phi_{0}, \theta_{0}\right) & \mathbf{a}_{H}\left(\phi_{1}, \theta_{1}\right) & \cdots & \mathbf{a}_{H}\left(\phi_{M-1}, \theta_{M-1}\right)
\end{array}\right]
$$

\section{AMBIGUITIES}

In DOA estimation of narrowband signals in antenna arrays applications there are ambiguities appearing as a few identical responses of antenna array to distinct coordinates of signal sources. A well known example of ambiguities can be seen in linear antenna arrays and as a consequence several additional false peaks arise in a spatial spectrum. The problem of ambiguities greatly impacts on practical using and implementing DOA-estimation methods. In application of antenna arrays for wireless communication systems the question can be asked: how to design an antenna array according to specified performances of resolution and accuracy of DOA-estimation and without ambiguities (without false peaks). Another problem is how to predict false peaks arising in the spatial spectrum, which are caused by ambiguities of an antenna array. The problem can be developed and formulated: how to separate antenna ambiguities from those specific for DOA estimation algorithms. As we know, linear antenna arrays have ambiguities in two planes (such arrays have spatial spectrum peaks which are symmetric about the central axis). For linear antenna the ambiguities of this kind can be easily predicted [15]. The problem gets more complicated for more complex geometries of antenna arrays.

Due to Schmidt [11], ambiguity I occurs when $\mathbf{a}\left(\phi_{i}, \theta_{i}\right)=\mathbf{a}\left(\phi_{j}, \theta_{j}\right), i \neq j$. Ambiguity I characterizes the situation with one incident signal on the antenna array. From the geometrical viewpoint, ambiguity I occurs, when in the array manifold in M-dimensional space the steering vector (one or more) collinear with the incident signal steering vector arises. The incident signal steering vector is in theory perfectly orthogonal to the noise subspace and a great number of subspace based DOA estimation algorithms (such as MUSIC) reckon on. In the case of one incident signal, the incident signal steering vector spans the signal subspace the dimensionality of which is equal to one. The steering vectors in the arrays manifold which are collinear with the incident signal steering vector but which do not correspond to the actual DOA are also orthogonal to the noise subspace. So, false $\operatorname{peak}(\mathrm{s})$ in the spatial spectrum occurs due to ambiguity. But what happens when in the array manifold one or some steering vectors are not perfectly collinear to the incident signal vector. It is evident that they introduce high level sidelobes. The function characterizing ambiguity I may be formulated as [16]:

$$
\chi_{i j}^{I}\left(\phi_{i}, \theta_{i}, \phi_{j}, \theta_{j}\right)=\frac{\mathbf{a}\left(\phi_{i}, \theta_{i}\right) * \mathbf{a}\left(\phi_{j}, \theta_{j}\right)}{\left\|\mathbf{a}\left(\phi_{i}, \theta_{i}\right)\right\|\left\|\mathbf{a}\left(\phi_{j}, \theta_{j}\right)\right\|} .
$$

The function $\chi_{i j}^{I}\left(\phi_{i}, \theta_{i}, \phi_{j}, \theta_{j}\right)$ is complex-valued, but its absolute values range from $[0,1]$. Values 1 and 0 correspond to the perfect collinearity and absolute orthogonality of vectors $i$ and $j$ respectively.

Consider a couple of variants of circular antenna arrays. Let's assume, that we have CARs consisting out of six antenna elements with radiuses $r=1.25 \lambda$ and $r=0.5 \lambda$. Let's calculate the function $\chi_{i j}^{I}\left(\phi_{i}, \theta_{i}, \phi_{j}, \theta_{j}\right)$ for the circular array in range of azimuth angles $\theta=\left[-150^{\circ} ; 150^{\circ}\right]$ and elevation angle $\varphi=70^{\circ}$. From fig. 5a it is seen, that for CAR with radius $\mathrm{r}=1.25 \lambda$ there are several combinations of perfectly collinear vectors. For example, they are for $\varphi=70^{\circ}$ and $\theta=30^{\circ},-90^{\circ},-30^{\circ}, 90^{\circ},-150^{\circ}$ and $150^{\circ}$, those vectors have $\chi_{i j}^{I}$ equal to one. Let us compute the spatial spectrum via MUSIC method to validate this combination. From fig. $6 \mathrm{a}$ it is seen, the peaks positions of CAR with $r=1.25 \lambda$ coincides with the maximum values of the function $\chi_{i j}^{I}$. Additionally, another considered geometry of CAR with $r=0.5 \lambda$ has not the false peaks in its spatial spectrum (fig. 6b). It can be clarified by $\chi_{i j}^{I}$ from fig. $5 b$, where there are no additional high values. Moreover, false peaks do not occur in the spatial spectrum of the concentric antenna arrays. And we can say that the situation is identical to circular antenna arrays with $r=0.5 \lambda$.

Ambiguity II is characterized by multiple signal sources, so the ambiguities are important for mobile communication systems, when multiple signals simultaneously impinge onto the antenna array. Due to Schmidt [11], ambiguity II occurs when array manifold intersects the $M$ dimensional signal subspace Es more than $K$ times. In order to characterize type II ambiguity of arbitrary antenna array the ambiguity II function [17]:

$$
\chi_{i}^{I I}\left(\phi_{i}, \theta_{i}\right)=\frac{\mathbf{a}\left(\phi_{i}, \theta_{i}\right) * \mathbf{E}_{S} \mathbf{E}_{S}^{H} * \mathbf{a}\left(\phi_{i}, \theta_{i}\right)}{\left\|\mathbf{a}\left(\phi_{i}, \theta_{i}\right)\right\|} .
$$

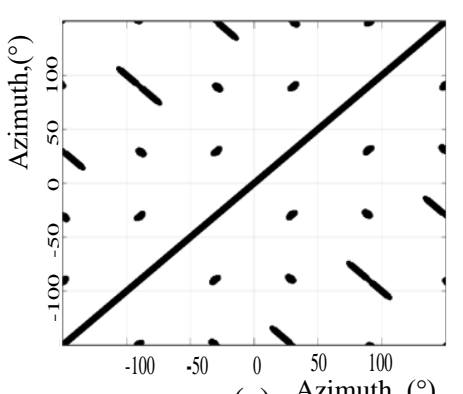

(a) Azimuth, $\left(^{\circ}\right)$

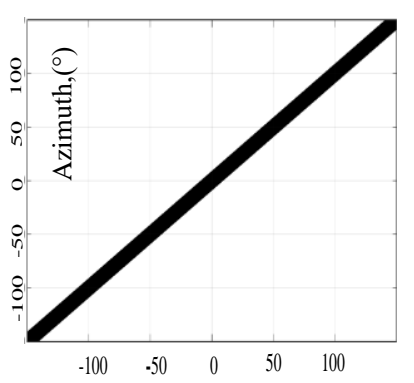

(b) Azimuth, $\left({ }^{\circ}\right)$
Fig. 5. Function $\chi_{i j}^{I}$ for CAR with radius a) $r=1.25 \lambda$ and b) $r=.5 \lambda$ 


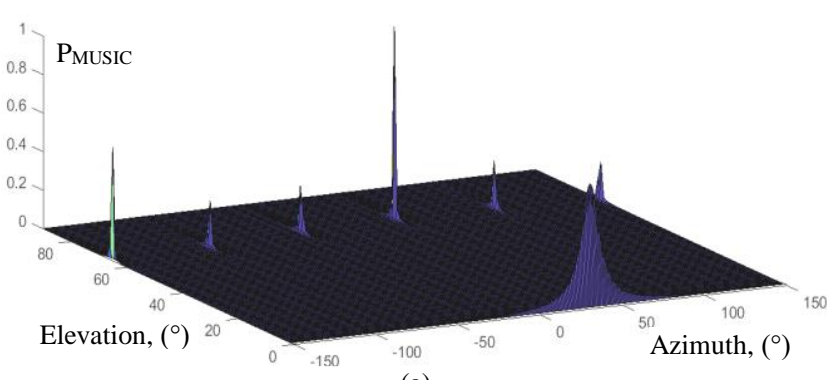

(a)

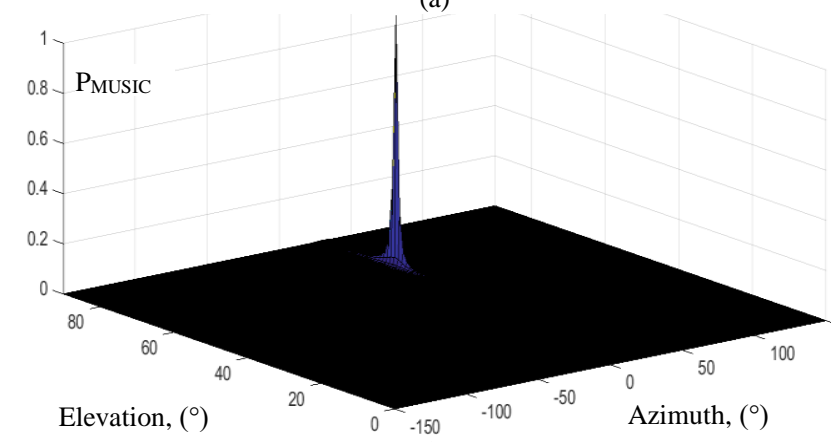

(b)

Fig. 6. Method MUSIC in the circular array of the radius a) $r=1.25 \lambda$, b) $r=0.5 \lambda$ when one signal arrives

Let's assume that a concentric antenna array consists of two circular antenna arrays of three and four antenna elements respectively. Those arrays have radiuses $0.1443 \lambda$ and $0.3536 \lambda$ respectively. Two waves arrive at the array with coordinates $\theta_{1}=20^{\circ}, \varphi_{1}=20^{\circ}$ and $\theta_{2}=45^{\circ}, \varphi_{2}=45^{\circ}$. From fig. $7 \mathrm{a}$ it is seen, there are multiple false peaks (the arrows point them) close around the actual coordinates of signal sources.

According to $\chi_{i}^{I I}\left(\phi_{i}, \theta_{i}\right)$ depicted in fig. $7 \mathrm{~b}$, the 'trace' of maximums stretches from the first coordinate to another. The levels of the false peaks close to an actual coordinate can exceed the ones close to another coordinate. That makes

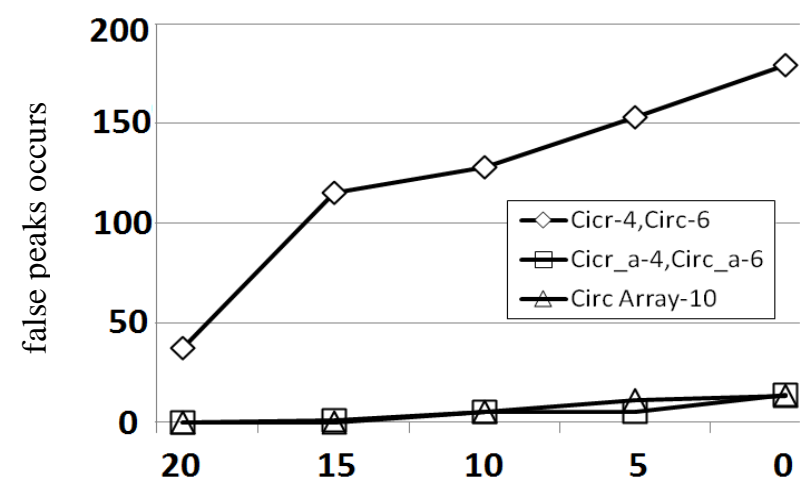

(a)

SNR, (dB)

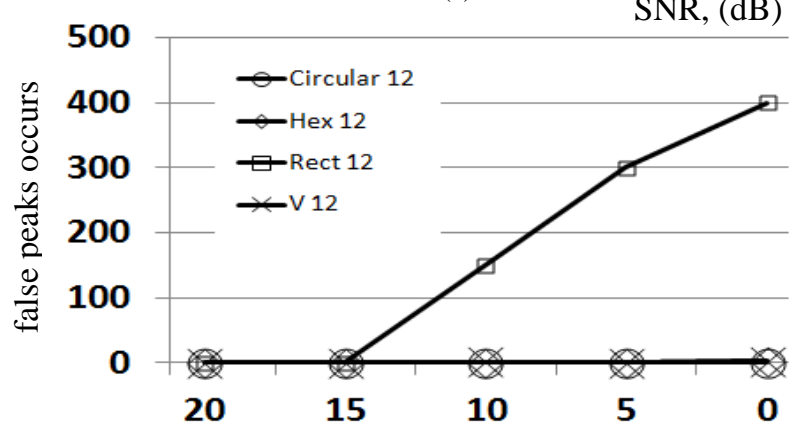

(c)

SNR, (dB)

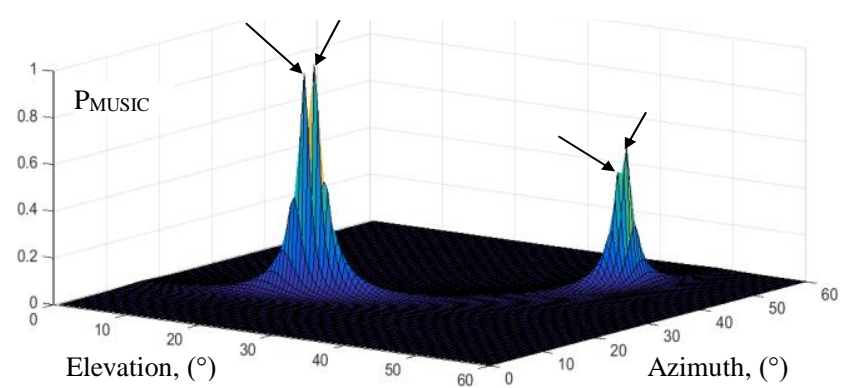

(a)

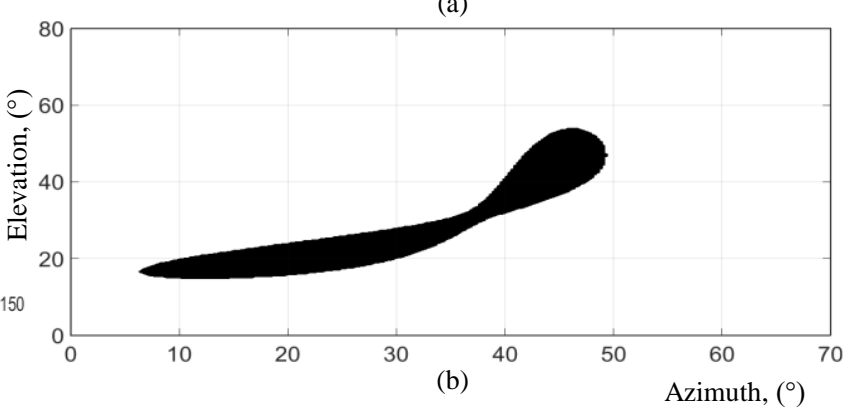

Fig. 7. a) Method MUSIC and b) function $\chi^{\mathrm{II}}$ for two signals in the circular array of the radius $r=0.5 \lambda$.

impossible to filter the false peaks on the level. Additionally, occurring false peaks and their levels are random.

Let us consider CAR of ten elements with inter-element distance $0.5 \lambda$ and radius $r=0.809 \lambda$ ('CircArray-10'). Additionally, we may regard the concentric antenna array with two CARs out of four and six antenna elements, interelement distance is $0.5 \lambda, r=0.1769 \lambda$ and $r=0.5 \lambda$ ('Circ-4, Circ-6'). We have simulated noise in the range of SNR -20 to $0 \mathrm{~dB}$ to measure statistically the probability of occurring false peaks. Two signals impinge at the arrays with coordinates $\theta_{1}=20^{\circ}, \varphi_{1}=20^{\circ}, \theta_{2}=45^{\circ}, \varphi_{2}=45^{\circ}$. In this experiment we try to measure how often the false peaks

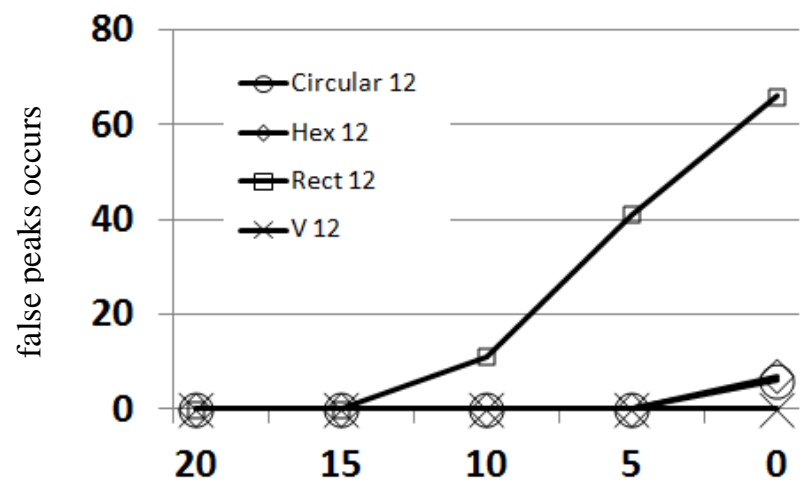

(b)

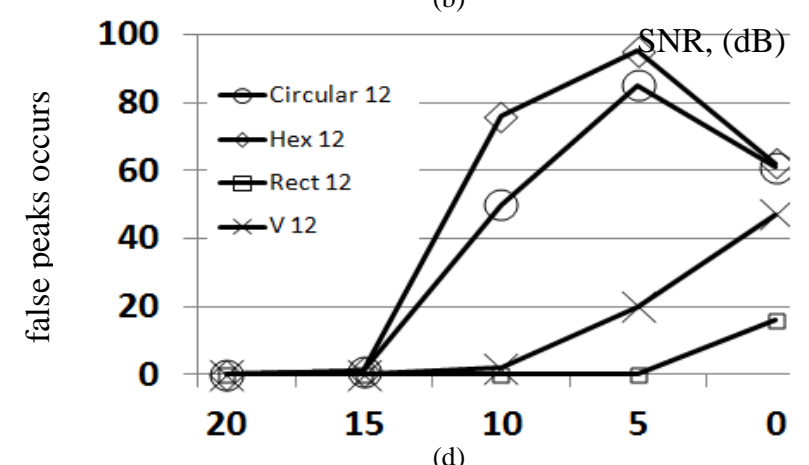

SNR, (dB)

Fig. 8. The number of false peaks occurrence in a) the circular and concentric antenna arrays for two signals from elevation angle $\varphi=45^{\circ}$, b) the arrays for two signals from elevation angle $\varphi=45^{\circ}$, c) the arrays for two signals from elevation angle $\varphi=80^{\circ}$ and d) the arrays for two signals from elevation angle $\varphi=30^{\circ}$ 

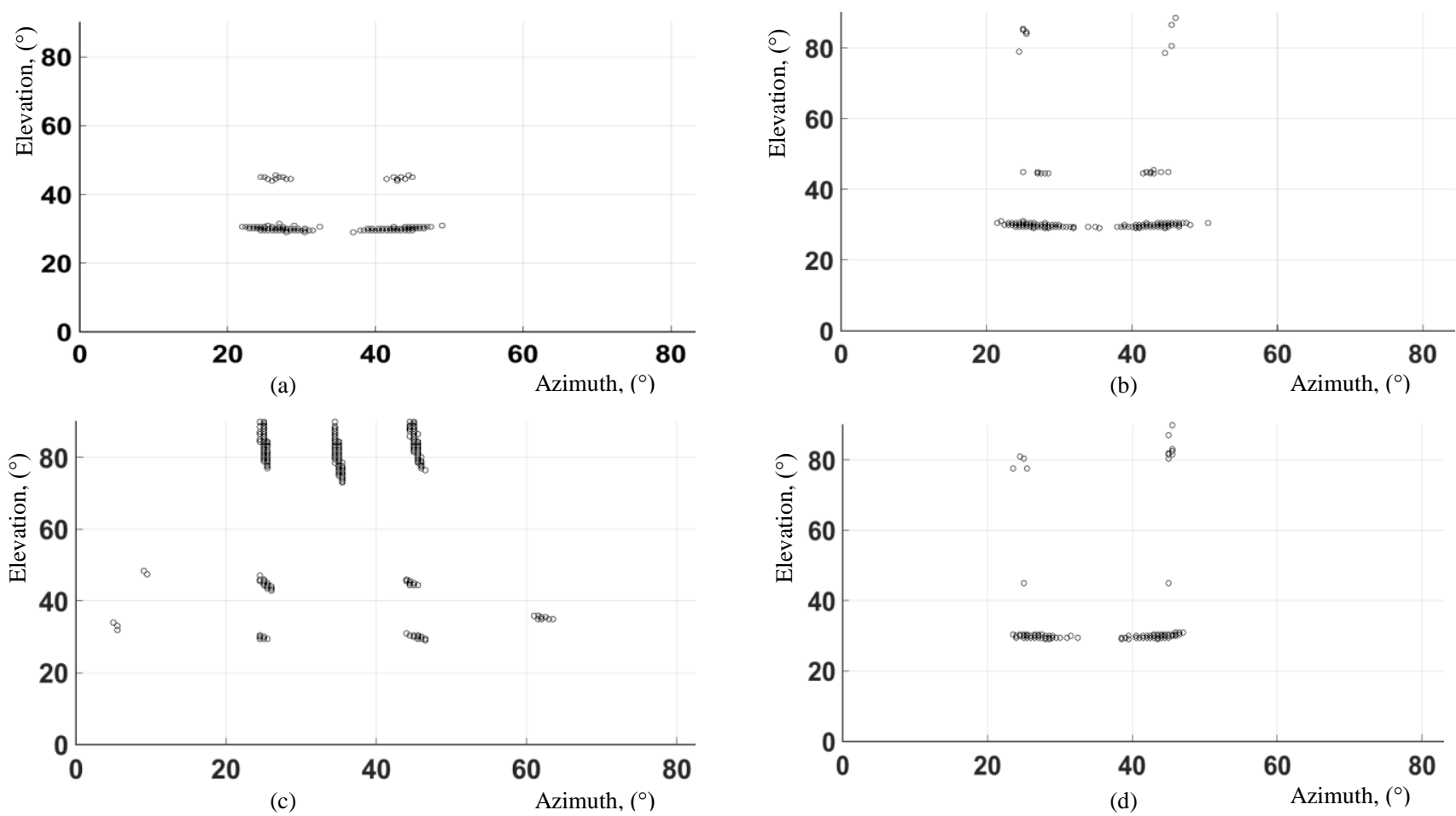

Fig. 9. False peaks distributions along spatial spectrum of MUSIC in a) Circular, b) Hexagonal, c) Rectangular and d) V-shaped antenna arrays

occur using different conditions and environments. While calculating the function (2), we increment the counter of the occurrence of false peaks in case it is possible to identify more peaks than the number of signals which is supposed to be known. The number of iterations is 500. As we can see from fig. $8 \mathrm{a}$ the number of false peaks occurring in the concentric antenna array DOA estimation by MUSIC is much higher than the value for the circular array. The difference is very large and reaches the value of about 100 . The behavior is related to decreased space of aperture of the concentric array with the same number of antenna elements. Consider the concentric antenna array with a radius of the outer circular antenna array equal to one of the considering circular array, that is $r=0.809 \lambda$ ('Circ_a-4, Circ_6'). It is seen from fig. $8 \mathrm{a}$ that the concentric arrays with the same aperture as the circular one have the comparable the number of false peaks occurrence. And the number is very small, about 10 .

Consider the most known and used forms of antenna arrays, i.e. in addition to circular; we take hexagonal, rectangular and V-shaped antenna arrays. All the considered antenna arrays have the equal number of antenna elements, placed equally along their perimeters, i.e. twelve elements. Each side of the hexagonal array and the rectangular array consist of two and three elements respectively. They are spaced between each other by $0.5 \lambda$. After making a few algebraic operations it turns out that the circumscribed circle of each antenna array has almost identical radius $r=(6 / 2 \pi) \lambda$. The V-shaped antenna array consists of two identical equispaced linear array with the common first element and an angle between them is equal to $45^{\circ}$. The range of the signal-noise ratio is from $20 \mathrm{~dB}$ up to $0 \mathrm{~dB}$, the number of the averaging samples $K$ of the spatial correlation matrix is 100 , the number of the iterations is 500 . Consider the results received after simulation of two arriving signals. It is a more interesting case because in a real application (radars, wireless radio, etc.) there are multiple signals arriving at antennas. Again the simulation is done for three elevation angles to learn the dependence of the number of false peaks occurrence on the position in space. Azimuth coordinates are $\theta_{1}=25^{\circ}, \theta_{2}=45^{\circ}$ for $\varphi=45^{\circ}, 80^{\circ}$ and $\varphi=30^{\circ}$. In Fig. $8 b-d$ the following names for antenna arrays are used: Circular 12- circular, Hex 12 - hexagonal, Rect 12 - rectangular, V $12-\mathrm{V}$-shaped. It is possible to make the several conclusions after viewing Fig. 8 b-d.

The rectangular antenna array is the one most prone to false peaks creation. It has the largest number of false peaks occurrence. From fig. $8 \mathrm{~b}-\mathrm{c}$ it is clearly seen that the rectangular configuration produces almost $100 \%$ probability out of 500 trials in the case of elevation angle $\varphi=80^{\circ}$ and higher than $10 \%$ in the case of signals position in the middle of elevation. Those numbers are much higher than the corresponding values of the other geometries.

It can be surely said that the antenna array form less prone to producing false peaks is a $\mathrm{V}$-shaped one. After viewing fig. $8 \mathrm{~b}$-d the $\mathrm{V}$-shaped antenna array of all the considered scenarios has the least amount of false peaks occurrence. Hexagonal and circular antenna arrays show similar numbers of all the cases. It should be noted that the signal position along $\mathrm{z}$ axis, which is the most prone to false peaks is close to $\varphi=0^{\circ}$. The causes of the decrease in the number of false peaks, when $\mathrm{SNR}=0 \mathrm{~dB}$ (case of circular and hexagonal antenna array) is under consideration.

Consider how the false peaks of the method MUSIC in the researched planar antenna arrays are distributed on the spatial chart. We will use the same conditions as used in the earlier experiment, i.e. the signal sources have the following coordinates: in first case $\theta_{1}=25^{\circ}, \varphi_{1}=30^{\circ}$ and $\theta_{2}=45^{\circ}$, $\varphi_{2}=30^{\circ}$; in second case $\theta_{1}=25^{\circ}, \varphi_{1}=45^{\circ}$ and $\theta_{2}=45^{\circ}, \varphi_{2}=45^{\circ}$; in third case $\theta_{1}=25^{\circ}, \varphi_{1}=80^{\circ}$ and $\theta_{2}=45^{\circ}, \varphi_{2}=80^{\circ}$. The peaks 
are displayed on the spatial plot if there are more than two peaks on the spatial spectrum of the MUSIC method in each separate case. In case there are only two peaks on the spatial spectrum of MUSIC, then these coordinates will not be depicted on the charts. In fig. 9 the false peaks coordinates are drawn for all the considered positions of the signal sources along the elevation plane and all the signal-noise ratios so as to give the whole picture.

As we can see from fig. 9, the rectangular antenna array generates the distributions of the false peaks coordinates which can be hard to identify and relate to one of the true coordinates. Their occurrences are rather random and hardly predictable. So it can be said that direction of arrival estimation of the signal coordinates can be hardly implemented with high accuracy.

If we consider the false peaks distributions generated by the rest of the antenna arrays, then it is obvious that their occurrence is basically treatable character and they lay in the vicinity of the true coordinates of the signals sources. These distributions can be easily processed by the clustering algorithms, for example $k$-means, after that inside each cluster a peak can be chosen with the highest value of the function (2) [18].

\section{CONCLUSION}

Planar antenna arrays, including circular, concentric and hexagonal, rectangular and V-shaped have been considered. These configurations allow estimating the directions of arrival of electromagnetic waves on azimuth and elevation planes. The math basis of the ambiguities of antenna arrays manifold has been considered as well. The phenomenon gives rise to several false peaks on the spatial spectrum along with the peaks pointing to actual signal coordinates. The probability of the occurring false peaks is measured by means of simulation of various noise environments. It is established that the more aperture, the less probability is. Additionally, the rectangular antenna array is the most prone to producing false peaks and V-shaped is the least. The rectangular antenna array's peaks are hard to process in order to estimate the signal sources coordinates. If the signal source is placed close to $0^{\circ}$ with respect to the $z$ axis or perpendicular to the plane of a planar antenna array, the probability of occurring false peaks is very high.

\section{REFERENCES}

[1] H. Krim, M. Viberg, "Two decades of array signal processing research," IEEE Signal Processing Magazine, Vol. 7, pp. 67-94, 1996.

[2] H.L. Trees Van, Detection, Estimation, and Modulation Theory, Optimum Array Processing. John Wiley \& Sons, 1470 p., 2002.
[3] Yu. B. Nechaev, S. A. Zotov, E. S. Makarov, "Correction of the amplitude-phase distribution of electromagnetic field in the context of wireless direction finding problem," Radioelectronics and Communications Systems, Vol.52, No.4, pp. 208-214, 2009.

[4] Yu. Nechaev, D. Borisov and I. Peshkov, "Estimating the accuracy of autocalibration methods for determining the coordinates of radio wave sources with a constant conditional model of amplitude and phase errors in a digital antenna array channels,' Telecommunications and Radio Engineering, Vol. 72, No. 11, pp. 945-958, 2013.

[5] Yu. Nechaev, I. Peshkov, "Evaluating Cramer-Rao Bound for 2D direction-finding via planar antenna arrays," Visn. NTUU KPI, Ser. Radioteh. radioaparatobuduv., No. 67, pp. 12-17. (in Russian)

[6] Hua Y., Sarkar T. K., and Weiner D. D. An L-shaped array for estimating 2-D directions of wave arrival. IEEE Trans. Antennas Propag., Vol. 44, pp. 889-895, Jun. 1996.

[7] H. Gazzah, S. Marcos, "Cramer-Rao bounds for antenna array design," IEEE Transactions on Signal Processing, Vol. 54, No. 1, Jan 2006, pp. $336-345$.

[8] S. Ozgur Ata and C. Isik, "High-Resolution Direction-of-Arrival Estimation via Concentric Circular Arrays," ISRN Signal Processing, Vol. 2013, Article ID 859590, 8 pages, 2013.

[9] Trees Van, H. L. Detection, Estimation, and Modulation Theory. Optimum Array Processing, John Wiley \& Sons, 2002, pp. 1470.

[10] L.C. Godara, "Applications of antenna arrays to mobile communications. Part I : performance improvement, feasibility, and system considerations," Proceedings of the IEEE, Vol. 85, No. 8, pp 1195-1245, 1997.

[11] R. Schmidt, "Multiple Emitter Location and Signal Parameter Estimation", IEEE Trans. On Ant. and Prop., Vol. AP-34, No. 3. 1986.

[12] P. Kumar, "Formulation and Synthesis of Hexagonal Prism Array Using Nature Inspired Algorithm," Master thesis, National Institute of Technology, Rourkela, India, 2014.

[13] T. Bird, Fundamentals of aperture antennas and arrays, John Wiley \& Sons, 2016.

[14] Y. Nechaev, I. Peshkov, "Accuracy Researching of Direction-ofArrival Estimation via Music for Circular, Octagonal, Hexagonal And Rectangular Antenna Arrays," International Journal of Research in Engineering and Science, Vol. 4, No. 9, September 2016, pp. 08-15.

[15] A. Manikas, C. Proukakis, "Modeling and Estimation of Ambiguities in Linear Arrays," IEEE Transactions on Signal Processing, Vol. 46 No. 8, pp.2166-2179, March 2001.

[16] M. Erik, M. Obradovic, "Ambiguity characterization of an arbitrary antenna arrays: type I ambiguity", Spread Spectrum Techniques and Applications, 1998. Proceedings., 1998 IEEE 5th International Symposium on, Vol. 2, pp. $399-403$.

[17] M. Erik, M. Obradovic, "Ambiguity characterization of an arbitrary antenna arrays: type II ambiguity," Spread Spectrum Techniques and Applications, 1998. Proceedings., 1998 IEEE 5th International Symposium on, Vol. 3, pp. 955 - 958.

[18] Y. Nechaev, I. Peshkov, Y.O. Aalmuttar Atheer, K.D. Al Khafaji Sarmad, "Accuracy evaluation of super-resolution doa estimation methods for ring and concentric antenna arrays," Radio communication theory and equipment, Vol. 1, No. 2, May 2016, pp $79-86$ 